\title{
Structural biomechanics of the
}

craniomaxillofacial skeleton under maximal

masticatory loading: Inferences and critical analysis based on a validated computational

\section{model}

\author{
Amir R. Pakdel, Cari M. Whyne, Jeffrey A. Fialkov \\ Version Post-print/Accepted Manuscript \\ Citation Pakdel AR, Whyne CM, Fialkov JA. Structural biomechanics of the \\ (published version) craniomaxillofacial skeleton under maximal masticatory loading: \\ Inferences and critical analysis based on a validated computational \\ model. J Plast Reconstr Aesthet Surg. 2017 Jun;70(6):842-850. doi: \\ 10.1016/j.bjps.2017.01.021 \\ Copyright/License

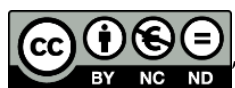 \\ BY NC ND This work is licensed under the Creative Commons \\ Attribution-NonCommercial-NoDerivatives 4.0 International License. \\ To view a copy of this license, visit Creative Commons NC BY ND 4.0 \\ License.
}

How to cite TSpace items

\begin{abstract}
Always cite the published version, so the author(s) will receive recognition through services that track citation counts, e.g. Scopus. If you need to cite the page number of the author manuscript from TSpace because you cannot access the published version, then cite the TSpace version in addition to the published version using the permanent URI (handle) found on the record page.
\end{abstract}

This article was made openly accessible by $U$ of $T$ Faculty. Please tell us how this access benefits you. Your story matters. 


\title{
Structural biomechanics of the craniomaxillofacial skeleton under maximal masticatory loading: inferences and critical analysis based on a validated computational model
}

\author{
Amirreza Pakdel, Cari Whyne, Jeffrey Fialkov
}

\begin{abstract}
Background: The trend towards optimizing stabilization of the craniomaxillofacial skeleton (CMFS) with the minimum amount of fixation required to achieve union, and away from maximizing rigidity, requires a quantitative understanding of craniomaxillofacial biomechanics.

This study uses computational modeling to quantify the structural biomechanics of the CMFS under maximal physiologic masticatory loading. Methods: Using an experimentally validated subject-specific finite element (FE) model of the CMFS, the patterns of stress and strain distribution as a result of physiological masticatory loading were calculated. The trajectories of the stresses were plotted to delineate compressive and tensile regimes over the entire CMFS volume.

Results: The lateral maxilla was found to be the primary vertical buttress under maximal bite force loading, with much smaller involvement of the naso-maxillary buttress. There was no evidence that the pterygo-maxillary region is a buttressing structure, counter to classical buttress theory. The stresses at the zygomatic sutures suggest that two-point fixation of zygomatic complex fractures may be sufficient for fixation under bite force loading.
\end{abstract}

Keywords: Zygomatic fracture; Computational model; Biomechanical models; Fracture fixation; Computer model validation; Craniofacial reconstructive surgery 


\section{Introduction}

The paradigm of facial skeletal stabilization for osteosynthesis has been shifting over the last few decades as a result of complications and morbidity arising from what can be termed "overengineering”. This idea refers to the maximization of mechanical stability following traumatic injuries or osteotomization of the craniomaxillofacial skeleton (CMFS) at the expense of biological preservation. This can result in a significant rate of CMFS fixation device removal $\left(84 \%^{1}, 20 \%{ }^{2}\right.$, $33 \%^{3}$ ), as well as complications that can be attributed directly to soft tissue disruption and compromised vascular supply inherent in the implementation of such fixation devices ${ }^{4}$.

There is an emerging shift towards minimizing both the amount of fixation required for adequate bony union ${ }^{5}$, and the degree of soft-tissue disruption and devascularization in order to lower complication rates ${ }^{4}$. For example, Champy et al. proposed using monocortical fixation on "ideal lines of osteosynthesis" as opposed to aiming for total rigidity with compression plating for mandibular angle fracture fixation ${ }^{6}$.

However, a critical question is remains: what are the "ideal lines of osteosynthesis" for the CMFS? The classical buttress theory is ubiquitously cited as the most fundamental biomechanical basis for skeletal stabilization. It is in large part based on Wolff's assumptions of form-function and patterns of bone density. Yet to date, the biomechanical basis of the buttress theory remains quantitatively unsubstantiated. The CMFS is difficult to model and analyze accurately owing to complexities in its structure (i.e. its intricate network of thin-cortical bones and physiologic loading



Computational modelling of the magnitude and distribution of stresses under physiologic loading is a tool that enables a rational and mechanistically rigorous approach to stabilizing compro- 
mised bony structures. Many clinically-relevant applications of computational biomechanics related to the CFMS are found in the literature ${ }^{11}$. However, direct experimental validation is vital for establishing the reliability and confidence in such models and their predictions ${ }^{12}$. The models also need to demonstrate reproducibility, with low sensitivity to inter-subject structural variability. Previous models in the CMFS literature have been limited both in validation and reproducibility ${ }^{13}$.

The current study aims to fill the above gap utilizing a new platform for patient-specific biomechanical modelling designed specifically for the $\mathrm{CMFS}^{7}$. This platform, incorporates novel image processing techniques that enable delineation of thin-bone morphology and high-resolution mapping of bone density and material properties from CT images. Subsequently the models generated were validated against experimental measurements on fresh-frozen cadaveric specimens mimicking unilateral single muscle (masseter and temporalis) loading scenarios. In the present study, based on these validated models, we developed and analyzed a comprehensive model of physiological biting. The resultant load transmission patterns are discussed in the context of clinical implications for facial skeletal stabilization. 


\section{Methods:}

\subsection{Finite element (FE) model generation and validation:}

A newly developed and experimentally validated method for subject-specific FE model generation was employed using deblurred clinical CT images, partial-volume correction, and high-

resolution material property mapping. The engineering details, presented in full elsewhere ${ }^{7}$, are described briefly here. A clinical CT scan of a fresh-frozen cadaveric CMFS specimen (mean age 86 years) was subjected to a full-stack 3D deblurring procedure by a PSF-based deconvolution method $^{14}$, which enabled visualization and segmentation of thin cortical structures. The CMFS volume was then segmented and meshed with $\sim 3$ million elements. Bone density values were mapped on to the model using an intensity-density relationship derived from bone density phantoms scanned within the CT data set. Bone properties were assigned in a subject-specific manner by high resolution mapping of densities derived from CT images to each node of each element. Subsequently, empirical conversion data was used to translate density values to elastic modulus values. 

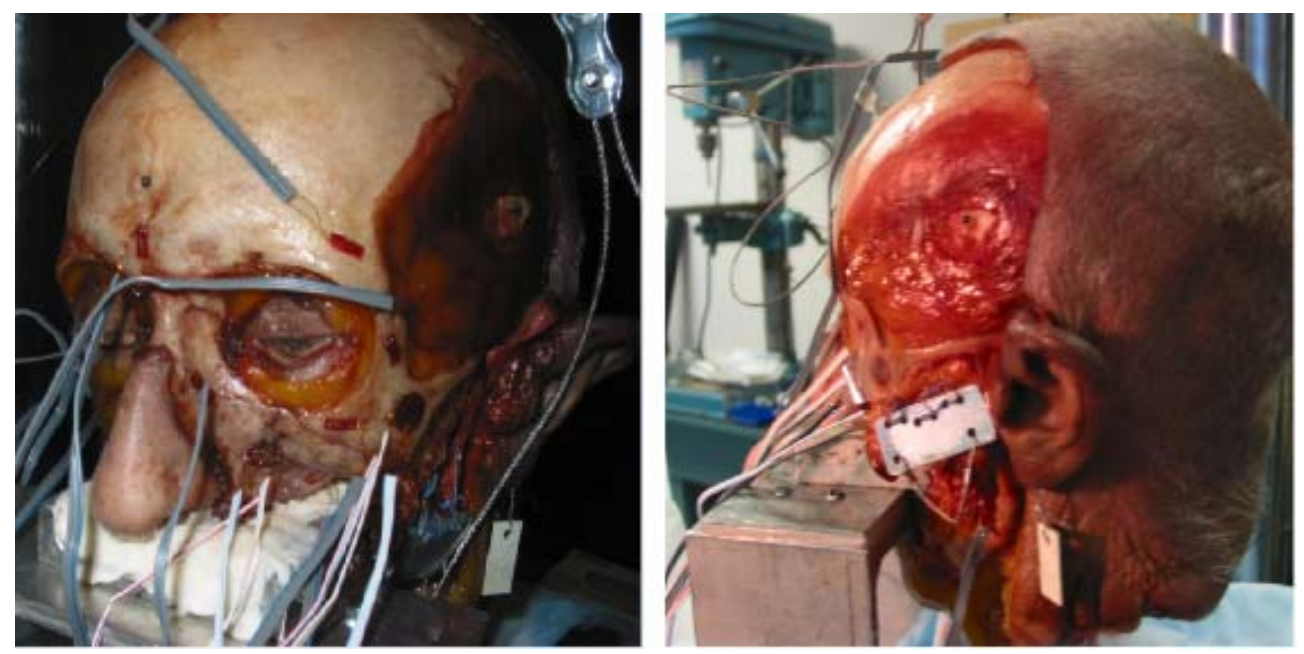

Figure 1. Experimental set-up for the in vitro characterization of surface strains due to masticatory muscle loading, used as in vitro data for validation of the strain predictions from the FE models

Experimental validation was conducted based on specimen specific in vitro mechanical testing (Figure 1). Loading was applied at multiple magnitudes individually through the masseter and temporalis muscles, with the maxillary occlusion fixed into bone cement. Strain gauges were affixed to the cortical surface of the CMFS region ipsilateral to the muscle loading. These loading boundary conditions at the muscle insertions and the displacement degree-of-freedom constraints at the occlusion were implemented into the FE model. A strong correlation was found in comparing the aggregate of strain values predicted across 5 FE models generated using this approach to those recorded from strain gauges from in vitro specimen specific experiments $(r=0.93$, slope $=1.01$, power $=0.81, \alpha=0.05)^{7}$.

\subsection{Simulation of physiological bite condition}

The previous step established the baseline reliability and reproducibility of the FE model for prediction of strain values. In the next step, new load and boundary conditions were applied to the validated FE model to simulate the physiological condition of maximal bite force loading. The mandible was segmented from CT scan of one of the male cadavers and repositioned to reflect 
clenched position. The superior surface of the condyle, the inferior surface of the glenoid fossa, and the mandibular and maxillary occlusal contact surfaces were delineated and brought into position guided by the articular tracking at the tempomandibular joint (TMJ) and an affine registration algorithm (Amira, Visage Imaging, San Diego, CA). The occlusion registration aligned the canines in close vertical proximity ( $3 \mathrm{~mm}$ separation) and placed the condyles in a position in close agreement with that reported in the literature ${ }^{15}$.

The CT images were used to localize the muscle insertions from the masseter, temporalis and medial pterygoid into the mandible. The masseter was inserted into the lower two-thirds of the ramus down to the mandibular angle, the temporalis inserted into the pyramidal surface of the coronoid process, and the medial pterygoid inserted into the medial aspect of the mandibular angle. The coordinates of these surface regions representing the muscle insertions were exported into the FE model bilaterally. The temporalis and masseter muscles were constructed as above and the regions of the muscle origins linked into the insertion zones on the original FE model. The origin of the medial pterygoid was chosen as those nodes within the pterygoid fossa and medial side of the lateral pterygoid plate (Figure 2).

Muscle force loading based on reported live subject values were applied bilaterally to the FE model, distributed over the insertion sites along a vector representing the average direction parallel to the muscle fibers ${ }^{16}$. The following loading, reported in the literature for a bite force of $200 \mathrm{~N}$, was applied: masseter $(120 \mathrm{~N})$, temporalis $(150 \mathrm{~N}$, including the anterior and posterior portions), and the medial pterygoids $(30 \mathrm{~N})$. All the degrees of freedom at the canine surface were set to zero. The nodes on the inferior surface of the glenoid fossa were assigned a sliding boundary 
condition, defined such that the displacement degree of freedom normal to the surface of the element for each node was zero (i.e. the boundary condition allowed sliding along the curvature of the fossa).

The distribution of stress patterns was reported as "trajectories" of tensile and compressive stresses in the CMFS model by computing the principal stress directions. These principal stress directions are either purely in tension or in compression (i.e. no shear). The major (first) and minor (third) principal stresses represent the largest tensile and the largest compressive stresses, respectively. A mapping of all of the major and minor principal stresses at all available points in the 3D model was used to produce a gross visualization of the trajectories of the tensile and compressive stresses.

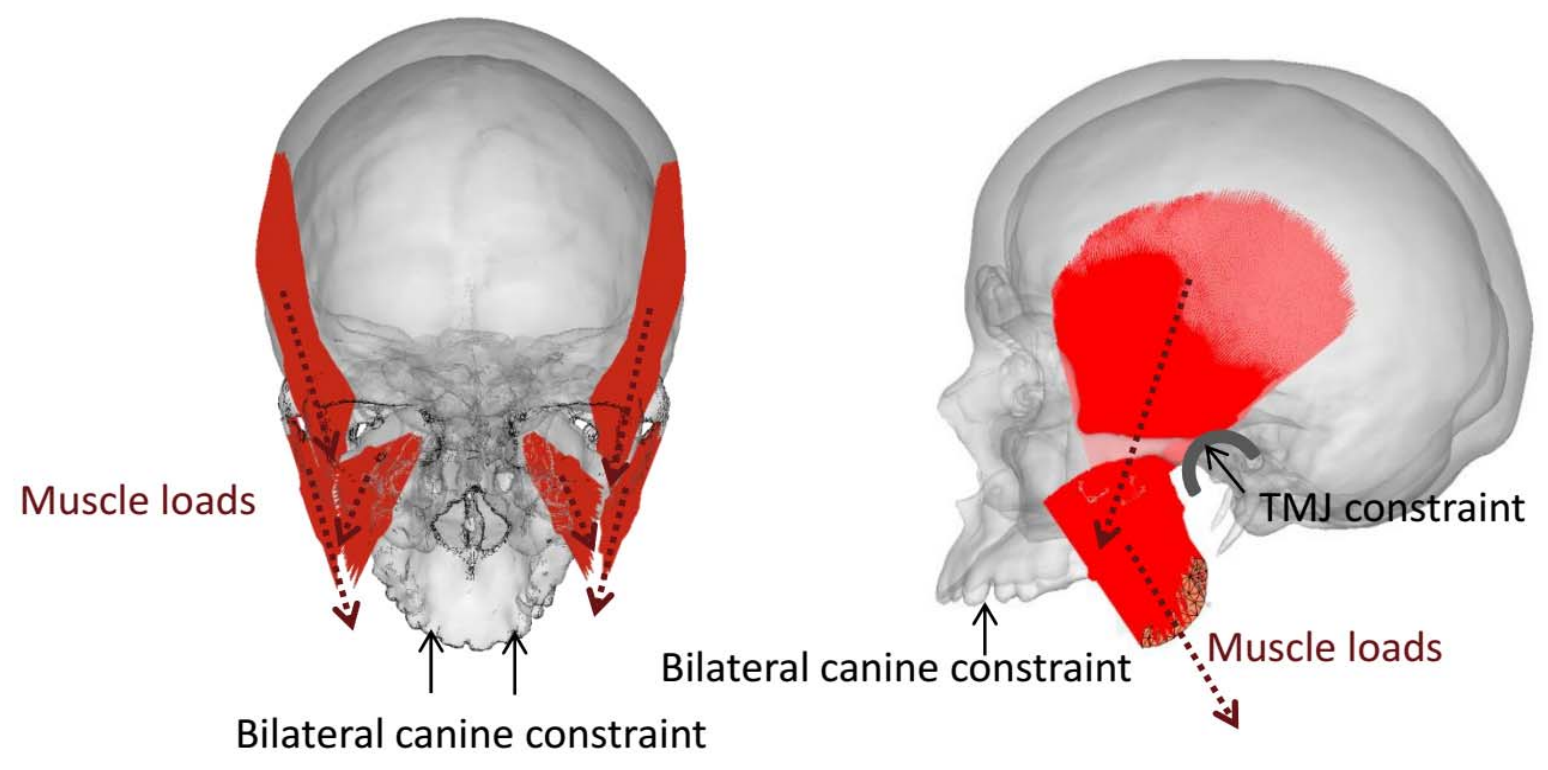




\section{Results}

The predicted bite force (total reaction force at the canines) and the TMJ joint reaction loads from the FE model under the applied muscle loading were $175 \mathrm{~N}$ and $171 \mathrm{~N}$, respectively. These results are within the range of published data by Rues et al. who reported a joint reaction load of $180 \pm 20 \mathrm{~N}$ under a bilateral canine bite force of $200 \mathrm{~N}$ in live subjects. ${ }^{16}$

The first principal (largest tensile) stress vectors are shown in Figure 3 on a positive color scale, and the third principal (largest compressive) stress vectors are plotted in Figure 4 using the same color scale, but inverted to depict the relative negative magnitudes. Slight bilateral asymmetry in the overall stress distribution pattern is evident. This is attributed to the subject's inclined occlusal plane. The tensile and compressive principal stresses within the maxillary and zygomatic bones are shown in isolation in Figure 5.

The salient features are summarized as follows:

The inferior orbital rim is in tension medially and in compression laterally, however the relative magnitude of these stresses is small.

The anterior medial and lateral maxilla is in compression while the posterior maxilla is not. The pterygo-maxillary "buttress" is purely under tension.

The inferior maxilla in the region of the molars is in compression. The anterior maxilla is also in compression, but has a compressive stress component that is directed posterolaterally.

The largest magnitude compressive loads are concentrated at the lateral aspect of the maxilla directed along the "lateral buttress" to the posteroinferior region of the zygoma. 
$\square \quad$ The pattern of tensile and compressive stress trajectories of the zygomatic arch indicate that it is under the action of compound moment arms.

o The temporal aspect of the arch, and its straight segment behave as a beam in bending, constrained at one end by the articular eminence.

o The medial and lateral walls of the zygomatic process of the arch have differential compressive-tensile trajectories, which indicates that this region of the arch is a vertically oriented plate in bending. 

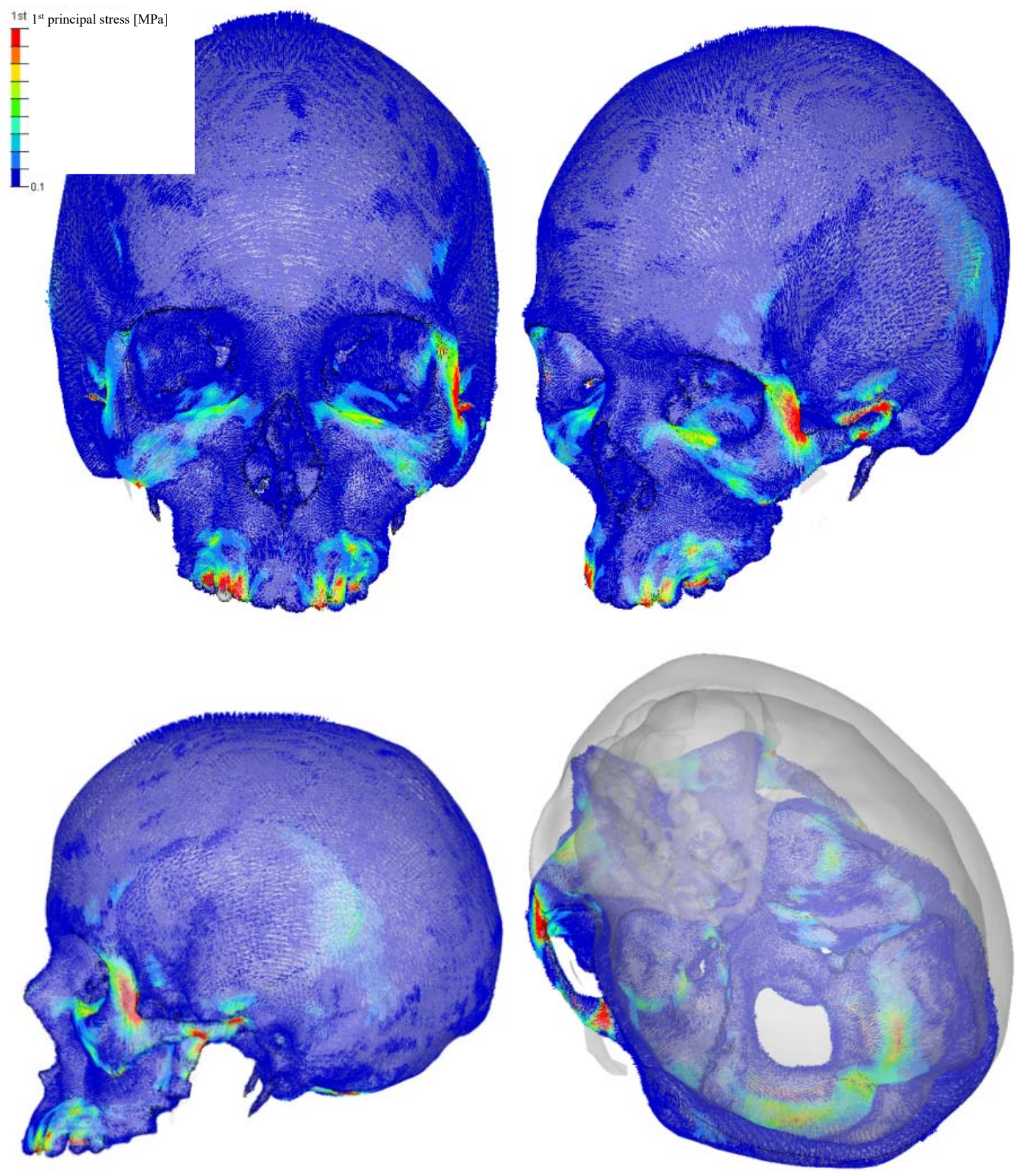

Figure 3. Plot of the highest invariant stress tensor, (the major principal stress) on the surface of the model (MPa), subject to the described biting condition (i.e. canine bite). The cross section in the lower right reveals the same stresses on the skull base. This vector represents the trajectory of stresses which are strictly in tension. They may be viewed as stresses which are "distracting" the bone under the applied loading condition. 

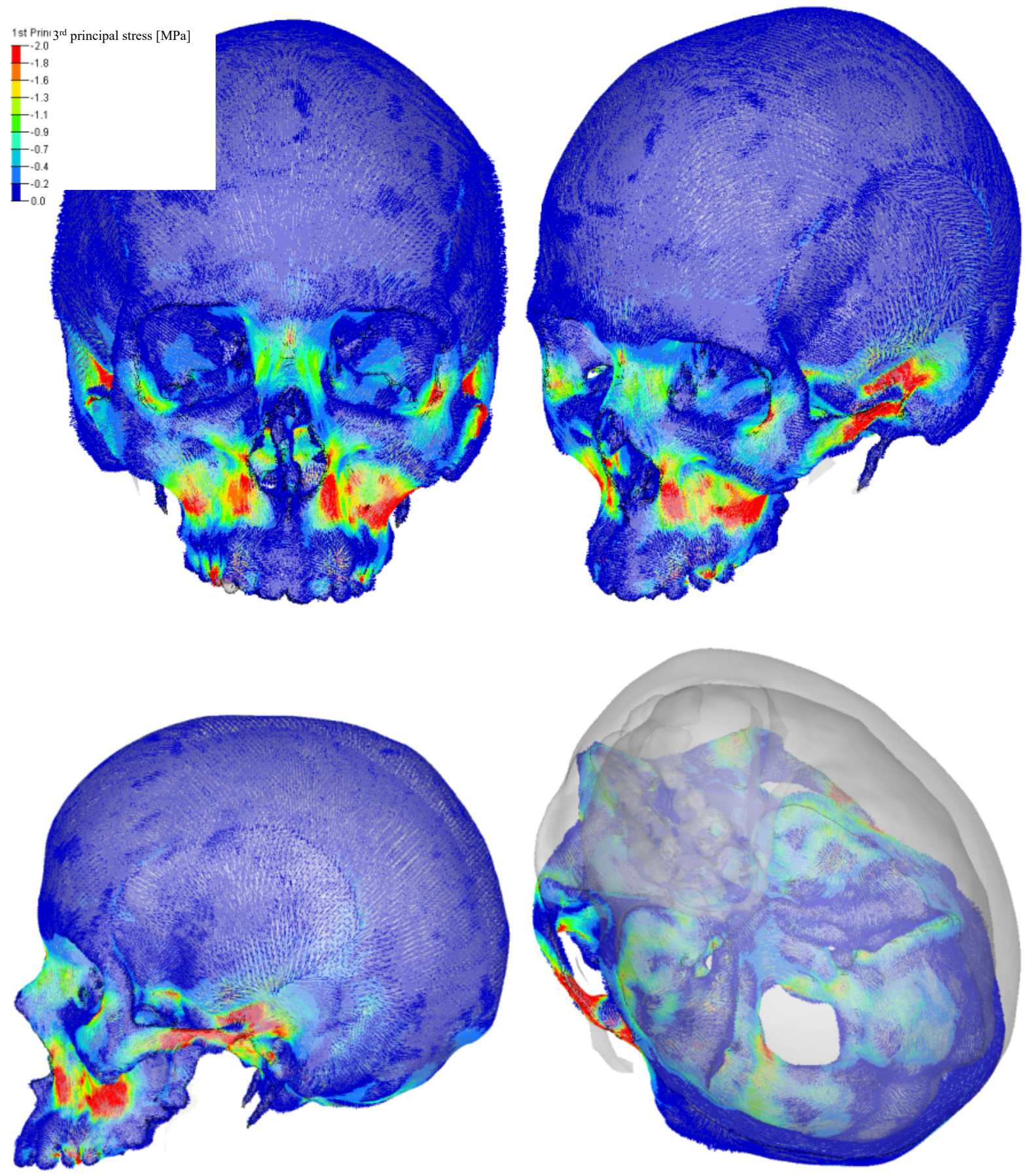

Figure 4. Plot of the most negative invariant stress tensors (the minor principal stress). The trajectories of this vector represent compressed structural regimes under the described given loading conditions (i.e. canine bite). 

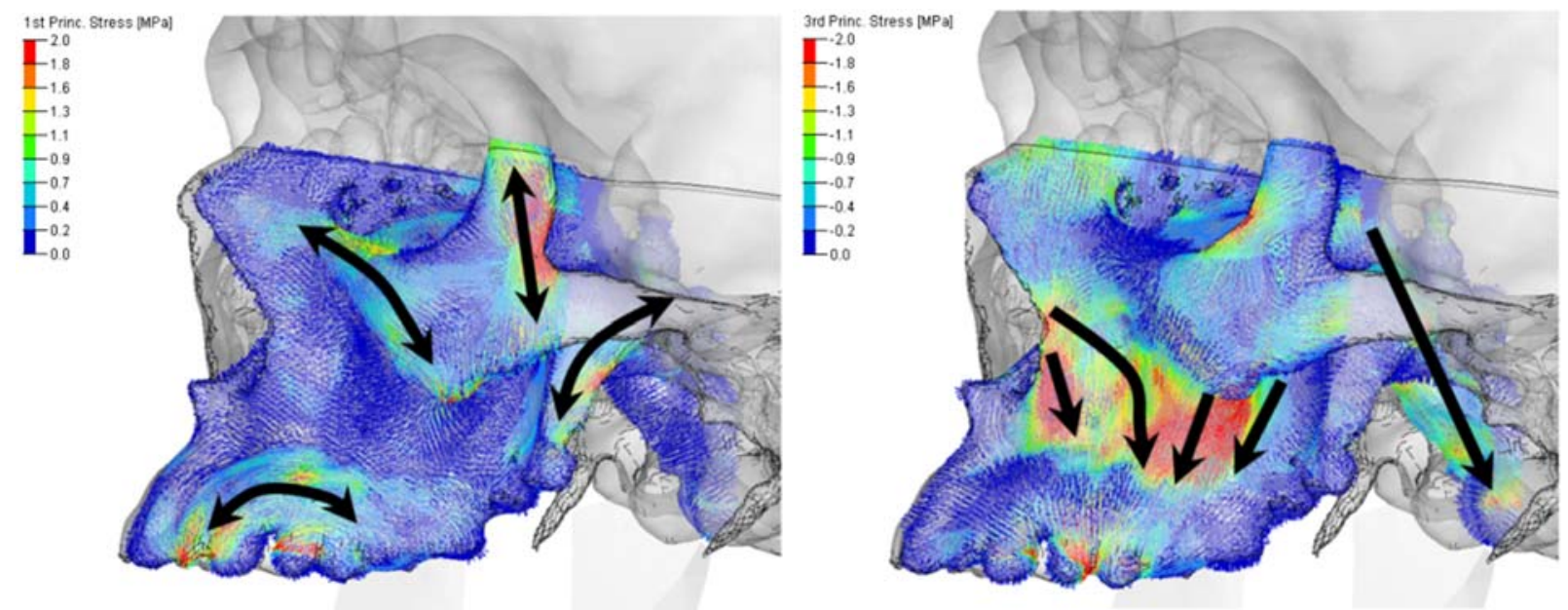

Figure 5. The tensile (left) and compressive (right) regions of the CMFS under the bite force loading as determined from the validated FE model.
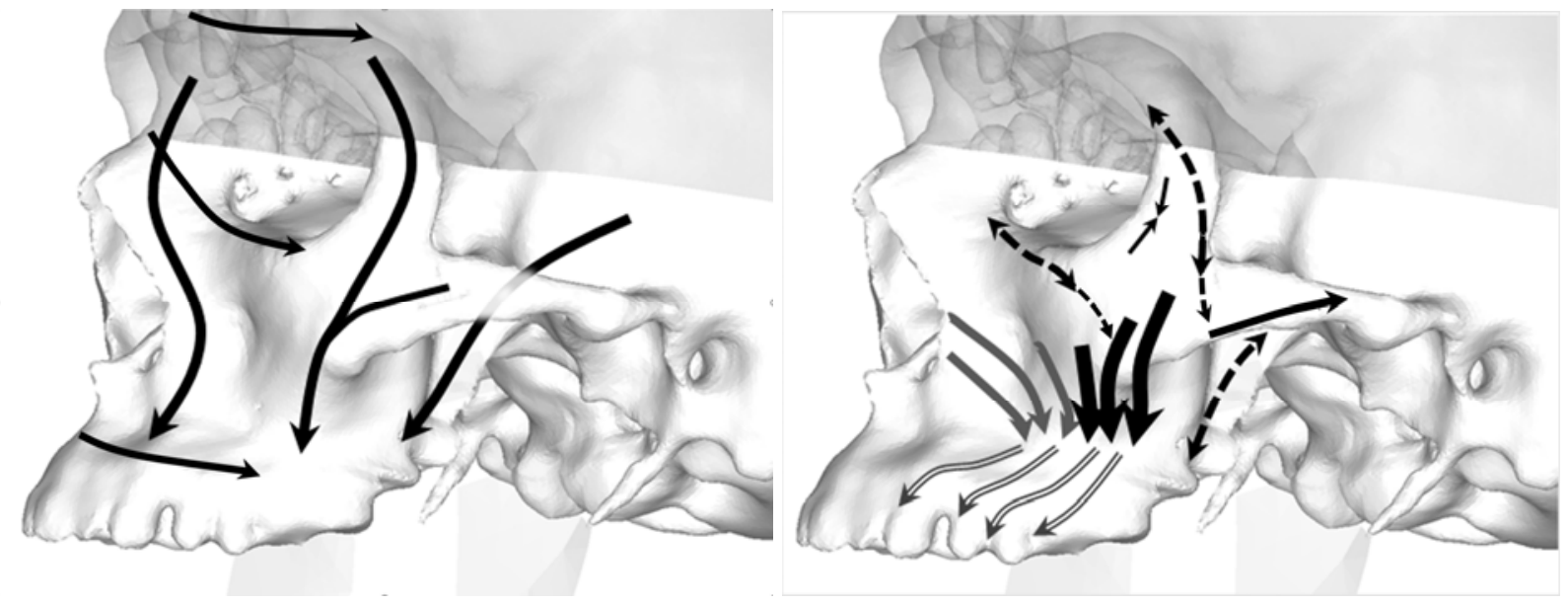

Figure 6. The left image depicts the proposed vertical load bearing struts of the CMFS as described by the classic buttress theory (from left to right: the naso-frontal, zygomatic-naso-frontal, and the pterygo-maxillary buttresses). The right image demonstrates the compressive load bearing structures (solid downward arrows) and the tensile trusses as revealed by the computational FE model under bite force loading (see Figure 5). 


\section{Discussion}

The principles of osteosynthesis for the fractured or osteotomized CMFS are changing based on the empirical observation that maximal rigid fixation may not be necessary to achieve bone healing and may in fact be a source of added morbidity due to biological and in particular vascular disruption. Classically, rigid fixation has been based on the buttress theory which makes the assumption that form determines function ${ }^{17-20}$.

The predicted bite forces and the joint reaction forces from the in vitro validated FE model yielded a close agreement to the in vivo EMG-based data and calculations by Rues et al. in the case of a maximal canine bite condition. As such, the continuum strain and stress patterns generated within the model may be used to better elucidate and understand the structural behavior of the CMFS. Some salient features (discussed below) of the observed stress distributions and their potential clinical implications for osteosynthetic stabilization run counter to classical buttress theory and assumptions held about CMFS biomechanics. This suggests that a validated accurate computational model has potentially disruptive implications to clinical practice in the field of CMFS reconstruction.

\subsection{The lateral maxilla is the primary vertical buttress of the CMFS}

Figure 6 illustrates how the computed pattern of stresses under masticatory loading contrasts with the classic buttress hypothesis. The majority of the vertical load is transmitted laterally through the maxilla, much more so than the magnitude observed at the naso-maxillary buttress. The trajectory of stresses in the pterygo-maxillary region were unexpected; the principal stress was strictly tensile in the pterygoid spines and posterior maxilla, suggesting that these structures mostly counter a torque produced in the more anterior structures. This is contrary to the expectation that 
the trajectory of forces in the pterygo-maxillary region should be compressive, if they are buttressing structures. This pattern of tensile stresses posterior to the maxilla correlates with the clinical observation of anterior open bite malocclusion seen in untreated Le Fort pattern maxillary fractures $^{21}$; The posterior maxilla experiences tensile stresses while the remainder of the maxilla is in vertical compression, resulting in posterior elongation and premature posterior contact. The constellation of these patterns suggest that the lateral maxillary buttress is the fundamental compressive load bearing structure of the midfacial CMFS in this bite scenario, and the other assumed buttresses either play only a minor role in countering vertical compressive bite forces, or are not countering compressive loads at all.

\subsection{Decoding the biomechanics of the zygomatic complex}

The zygomatic arch is a unique feature of the CMFS; its distended shape and isolated role as the attachment for the masseter suggest that it has a specialized structural role. The pattern of tensile and compressive stress trajectories indicate that it is under the action of compound moment arms under bite force loading, and cannot be described as behaving as a simplified structural unit (such as a truss or beam). The compound bending and torque patterns found on the zygomatic arch have been previously hinted at in data from in vivo studies of primates ${ }^{22}$ and observations in the posterior portion of the arch in pigs $^{23}$. Recorded strains on the zygomatic arch have shown tensile strains on its lateral surface and compressive strains on its medial surface ${ }^{24}$, in alignment with the general patterns described in the results section. The strain patterns presented substantiate the biomechanical basis for the tendency of the fractured arch to bow out laterally and the critical technical step of ensuring that it is flat during fixation.

The lateral orbital rim of the zygoma does not transmit compressive stresses to the frontal bone in a manner of a buttress. The sutural articulation at the frontal bone appears as a rigid constraint 
boundary for this region of the zygoma. This is an example where the function of a skeletal structure may run counters to heuristic judgments inferred from its outward morphology. The pillar-like appearance of this region of the zygoma suggests that it is adapted to bear compressive vertically oriented loads under masticatory stress ${ }^{26}$. However, the computational model provides evidence that even under maximal bite force loading this region is a beam in bending. It may be hypothesized that the zygomatic process of the frontal bone represents a distinct functional boundary which isolates structures adapted for masticatory function from the remainder of the skull. These results corroborate those of Oyen et al. who measured in vivo strains on the lateral orbital rim of African green monkeys (Cercopithecus africanus) and recorded only tensile stresses under biting loading conditions $^{27}$.

\subsection{Clinical relevance of the computational result to the treatment of}

\section{zygomatic complex fractures}

There is still no consensus on the optimal strategy for treating zygomatic complex fractures ${ }^{28-}$

${ }^{30}$. Some clinicians recommend maximal rigid three point fixation for best outcomes and attribute relapses to lack of adequate fixation to withstand masticatory loading ${ }^{31,32}$. Yet others report satisfactory outcomes with two or even one-point fixation ${ }^{33-35}$. Mechanical experiments have shown that the load to failure of a rigidly fixed zygomatic fracture is independent of the stiffness or strength of the fixation materials ${ }^{36}$. These suggest that maximal rigidity and strength are not required to achieve reliable bone healing. Applying multiple fixation devices requiring more dissection with greater biological disruption (inherent in the drilling and compressive application of the hardware) may not only be superfluous, it may worsen outcomes. 
Confirmation of vector and relative magnitudes of the principle tensile stress at the lateral orbital rim and compressive stress at the lateral maxillo-zygomatic buttress may help guide and optimize fixation requirements at these sites. For this common fracture pattern, minimal fixation directed along the axis of the tensile principal stress at the lateral orbital rim, and stabilization along the axis of the compressive principal stress in accordance with load bearing requirements at the lateral buttress appear to be indicated. The implication of the latter is that in non-comminuted fractures at the maxillozygomatic site, in which the bone is adequately buttressed, smaller plates and lesser fixation may suffice (e.g. to withstand shear displacement).

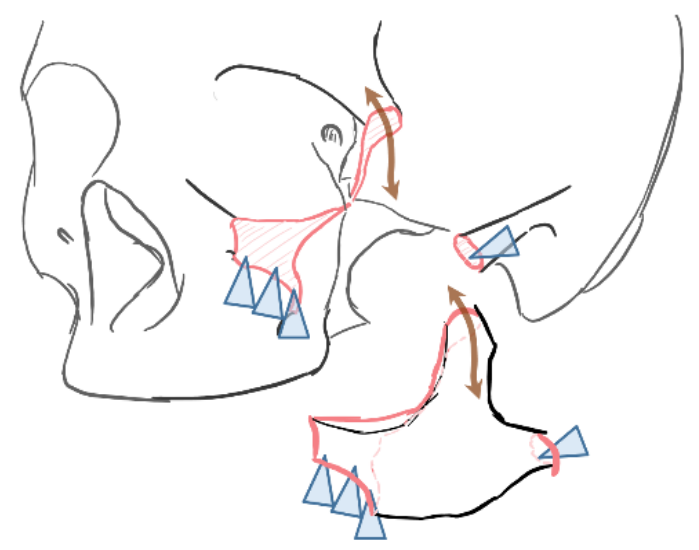

Figure 7. Simplified schematic of the directionality of the forces acting on the zygoma, based on the trajectory of stresses calculated for the maximal bite loading analyzed in this study. The blue triangles represent compressive forces. The brown arrows demonstrate distracting/tensile forces.

Based on the observed patterns of minimal stress at the inferior orbital rim, maximal tension at the lateral orbital rim and maximal compression at the lateral zygomatico-maxillary buttress, plating of the inferior orbital rim in zygomatic complex fractures may be redundant. Exposure and fixation of the inferior orbital rim fracture site through lower lid access, is associated with a significant amount of morbidity including hardware visibility, palpability, lid malposition, malar softtissue ptosis, etc. Yet, the magnitude of stresses along this rim is small and significantly lower than 
the tensile stress seen at the lateral orbital rim, which also has an appreciably greater area. As has been previously suggested based on empirical observation, the indication for extending exposure should be dictated primarily by the need for improving the accuracy of the reduction and not for the maximization of fixation ${ }^{35}$. Consequently, adequate visualization of the inferior rim, when required for reduction, from the upper buccal sulcus incision may preclude the need for any lower lid incision in the absence of indications for orbital floor exploration, as this exposure appears not to contribute to better stabilization. The computational evidence therefore supports the previously asserted clinical observation that three point fixation is unnecessary to achieve stabilization ${ }^{37,38}$. Furthermore, it is apparent from the computational data that optimal placement of fixation devices would be at the zygomaticofrontal fracture site and at the lateral zygomaticmaxillary fracture site, obviating the need for a potentially morbid trans-palpebral inferior rim exposure.

\section{Conclusion}

The current paradigm shift in CMFS stabilization following traumatic injuries, based on clinical experience and empirical data, has motivated a better understanding of CMFS biomechanical behavior. Clear delineation of the minimal requirements for mechanical stabilization, based on an accurate prediction of stress and strain patterns, may limit "over-engineering" that can lead to added morbidity. Although limited to a single scenario of maximal static bite loading, this work demonstrates a functional and validated platform for a critical analysis of currently held concepts of CMFS mechanics. A rigorous and quantified approach provides a theoretical basis on which to assess practical new approaches to CMFS stabilization (i.e. minimal strain regions requiring no fixation thus limiting morbidity inherent in exposure and fixation). Quantification of the stress and strain patterns in the facial skeleton can guide a rational approach to clinical decision making, to 
determine the amount and configuration of fixation required to achieve reliable healing and minimize the biological disruption.

The presented model attempts to bring us closer to this ideal. As a rigorously validated computational representation of the mechanical environment of the CMFS, it serves as a theoretical platform for critical analysis of current concepts of CMFS mechanics. By demonstrating correlation with clinical observations and experimental data under physiologic loading, the current model may prove to be a practical tool for the analysis of current practice techniques as well as the development of design criteria for future technologies for the treatment of CMFS injury and disease. Future work will focus on modeling pathological case scenarios such as the fractured or dysmorphic facial skeleton, as well as the effect of fixation devices on force distribution in these scenarios.

\section{Conflict of interest None.}

Acknowledgments Financial support for this work was provided by the Natural Sciences and Engineering Research Council of Canada (NSERC \#RGPIN239206-11), and the Ontario Graduate Scholarship Program. 


\section{References:}

1. Kubota, Y. et al. Association between plate location and plate removal following facial fracture repair. J. Plast. Reconstr. Aesthet. Surg. 65, 372-8 (2012).

2. Thorén, H. et al. Symptomatic plate removal after treatment of facial fractures. J. Craniomaxillofac. Surg. 38, 505-10 (2010).

3. Nagase, D. Y., Courtemanche, D. J. \& Peters, D. A. Plate Removal in Traumatic Facial Fractures. Ann. Plast. Surg. 55, 608-611 (2005).

4. Regev, E., Shiff, J. S., Kiss, A. \& Fialkov, J. a. Internal fixation of mandibular angle fractures: a meta-analysis. Plast. Reconstr. Surg. 125, 1753-60 (2010).

5. Rudderman, R. H. \& Mullen, R. L. Biomechanics of the facial skeleton. Clin. Plast. Surg. 19, 11-29 (1992).

6. Champy, M., Lodde, J. P., Jaeger, J. H. \& Wilk, A. Mandibular osteosynthesis according to the Michelet technic. I. Biomechanical bases. Rev. Stomatol. Chir. Maxillofac. 77, 56976

7. Pakdel, A., Fialkov, J. \& Whyne, C. High resolution bone material property assignment yields robust subject specific finite element models of complex thin bone structures. $J$. Biomech. In press, (2016).

8. Pakdel, A., Mainprize, J. G., Robert, N., Fialkov, J. \& Whyne, C. M. Model-based PSF and MTF estimation and validation from skeletal clinical CT images. Med. Phys. 41, 011906 (2014). 
9. Pakdel, A., Robert, N., Fialkov, J., Maloul, A. \& Whyne, C. Generalized method for computation of true thickness and x-ray intensity information in highly blurred sub-millimeter bone features in clinical CT images. Phys. Med. Biol. 57, 8099-116 (2012).

10. Szwedowski, T. D., Whyne, C. M. \& Fialkov, J. a. Toward characterization of craniofacial biomechanics. J. Craniofac. Surg. 21, 202-7 (2010).

11. Hannam, a G. Current computational modelling trends in craniomandibular biomechanics and their clinical implications. J. Oral Rehabil. 38, 217-34 (2011).

12. Erdemir, A., Guess, T. M., Halloran, J., Tadepalli, S. C. \& Morrison, T. M. Considerations for reporting finite element analysis studies in biomechanics. J. Biomech. 45, 625-33 (2012).

13. Trabelsi, N., Yosibash, Z., Wutte, C., Augat, P. \& Eberle, S. Patient-specific finite element analysis of the human femur--a double-blinded biomechanical validation. J. Biomech. 44, $1666-72(2011)$

14. Pakdel, A., Hardisty, M., Fialkov, J. \& Whyne, C. Restoration of thickness, density, and volume for highly blurred thin cortical bones in clinical CT images. Ann. Biomed. Eng. In press, (2016).

15. Yang, H. J., Kim, D. S., Yi, W.-J. \& Hwang, S. J. Reduced joint distance during TMJ movement in the posterior condylar position. J. Craniomaxillofac. Surg. 41, e159-64 (2013).

16. Rues, S., Lenz, J., Türp, J. C., Schweizerhof, K. \& Schindler, H. J. Muscle and joint forces under variable equilibrium states of the mandible. Clin. Oral Investig. 15, 737-47 (2011).

17. Pollock, R. A. Craniomaxillofacial Buttresses - Anatomy and Operative Repair. Head \& 
Neck 35, (Thieme, 2012).

18. Manson, P. N., Hoopes, J. E. \& Su, C. T. Structural pillars of the facial skeleton: an approach to the management of Le Fort fractures. Plast. Reconstr. Surg. 66, 54-62 (1980).

19. Kelly, K. \& Manson, P. Sequencing LeFort fracture treatment (Organization of treatment for a panfacial fracture). J. Craniofac. Surg. (1990).

20. Sicher, H. \& Tandler, J. Anatomie für zahnärzte. (J. Springer, 1928).

21. Miloro, M. \& Kolokythas, A. Management of Complications in Oral and Maxillofacial Surgery. (Wiley, 2011).

22. Hylander, W. L. \& Johnson, K. R. In vivo bone strain patterns in the zygomatic arch of macaques and the significance of these patterns for functional interpretations of craniofacial form. Am. J. Phys. Anthropol. 102, 203-32 (1997).

23. Rafferty, K. L., Herring, S. W. \& Artese, F. Three-dimensional loading and growth of the zygomatic arch. J. Exp. Biol. 203, 2093-104 (2000).

24. Oberheim, M. C. \& Mao, J. J. Bone strain patterns of the zygomatic complex in response to simulated orthopedic forces. J. Dent. Res. 81, 608-12 (2002).

25. Gart, M. S. \& Gosain, A. K. Evidence-Based Medicine. Plast. Reconstr. Surg. 134, 13451355 (2014).

26. Hardt, N. Craniofacial Trauma. (Springer Berlin Heidelberg, 2010). doi:10.1007/978-3$540-33041-7$

27. Oyen, O. J., Melugin, M. B. \& Indresano, A. T. Strain gauge analysis of the frontozygomatic region of the zygomatic complex. J. Oral Maxillofac. Surg. 54, 1092-1095 (1996). 
28. Barry, C. P., Ryan, W. J. \& Stassen, L. F. a. Anatomical study of factors contributing to zygomatic complex fracture instability in human cadavers. Plast. Reconstr. Surg. 119, 637-40; discussion 641 (2007).

29. Maturo, S. \& Lopez, M. a. Zygomatico-orbito-maxillary complex fractures. Oper. Tech. Otolaryngol. Neck Surg. 19, 86-89 (2008).

30. Eski, M. et al. A retrospective analysis of 101 zygomatico-orbital fractures. J. Craniofac. Surg. 17, 1059-64 (2006).

31. Candamourty, R. \& Narayanan, V. Treatment modalities in zygomatic complex fractures: A prospective short clinical study. Dent. Med. Res. 1, (2013).

32. Rana, M. et al. Surgical treatment of zygomatic bone fracture using two points fixation versus three point fixation--a randomised prospective clinical trial. Trials 13, 36 (2012).

33. Kovács, a F. \& Ghahremani, M. Minimization of zygomatic complex fracture treatment. Int. J. Oral Maxillofac. Surg. 30, 380-3 (2001).

34. Haerle, F. \& Champy, M. Atlas of Craniomaxillofacial Osteosynthesis: Microplates, Miniplates, and Screws. (Thieme, 2011).

35. Chakranarayan, A., Thapliyal, G. K., Sinha, R. \& Suresh, M. P. Efficacy of two point rigid internal fixation in the management of zygomatic complex fracture. J. Maxillofac. Oral Surg. 8, 265-9 (2009).

36. Kasrai, L., Hearn, T., Gur, E. \& Forrest, C. R. A biomechanical analysis of the orbitozygomatic complex in human cadavers: examination of load sharing and failure patterns following fixation with titanium and bioresorbable plating systems. J. Craniofac. Surg. 10, 237-43 (1999). 
37. Ellis, E. \& Kittidumkerng, W. Analysis of treatment for isolated zygomaticomaxillary complex fractures. J. Oral Maxillofac. Surg. 54, 386-400 (1996).

38. Kim, S. T. et al. Comparison of 1-point fixation with 2-point fixation in treating tripod fractures of the zygoma. J. Oral Maxillofac. Surg. 69, 2848-2852 (2011). 